\title{
NUMBER AND VIABILITY OF PARASITE INFLUENCE SEIZURE FREQUENCY IN CHILDREN WITH NEUROCYSTICERCOSIS
}

\author{
Lisiane S. Ferreira ${ }^{1}$, Li M in Li², Verônica A. Zanardi*2, Marilisa M. Guerreiro² \\ ABSTRACT - We investigated the relationship between CT findings - number and the viability phase of the \\ parasites - and the seizure frequency in children with neurocysticercosis before and short-term after antiepileptic \\ drug (AED) introduction. We only found a significant interaction between stage of parasitic infection and \\ number of lesions on seizure frequency after AED treatment. Patients with more than five lesions on active or \\ transitional stages had higher seizure frequency predicting a worse short-term prognosis.
}

KEY WORDS: neurocysticercosis, epilepsy, childhood.

\begin{abstract}
Número e viabilidade do parasita influenciam a frequência de crises epilépticas em crianças com neurocisticercose

RESUMO - No presente estudo, investigou-se a relação entre os achados tomográficos - número e estágio de viabilidade dos parasitas - e a frequência de crises epilépticas em crianças com neurocisticercose antes e logo após a introdução do tratamento com drogas antiepilépticas. Encontramos que a interação entre o estágio do parasita e o número de lesões influenciam significativamente a frequência de crises somente após o tratamento. Pacientes com mais do que cinco lesões em fase ativa ou transicional tiveram maior frequência de crises predizendo um pior prognóstico.
\end{abstract}

PALAVRAS-CHAVE: neurocisticercose, epilepsia, infância.

Neurocysticercosis (NC) is a main health problem in developing countries. It affects patients of all ages and it is endemic among children as well as adults in Latin America ${ }^{1}$. It is also becoming increasingly common in developed countries due to migration of carriers from endemic areas.

Epileptic manifestations appear to depend on number and localization of the cysts ${ }^{2}$. Nevertheless, some authors have not found any difference in seizure frequency or in clinical characteristics between patients with a single and multiple lesions ${ }^{3}$.

The objective of this study was to investigate the relationship between $\mathrm{CT}$ findings - number and the viability phase of the parasites - and the seizure frequency in children with NC.

\section{METHOD}

This is a historic cohort study. We selected 28 epileptic patients less than 16 years with probable or definitive diagnosis of NC according to Del Bruto et al. ${ }^{4}$. Sixteen were girls, the mean age was 7.2 years, and the mean followup was 64.5 months. All patients had parenchymal form of NC and normal neurological examination.

We obtained all information on the 28 patients on revision of their medical records, complemented with direct interview with patients and guardians whenever possible.

A semi-structured protocol was filled in for every patient, considering: seizure frequency before AED introduction (total number of seizures was classified ${ }^{5}$ as: $1-3$ per year $=5$; 4-11 per year $=6 ; 1-3$ per month $=7 ; 1-6$ per week $=8 ; 1$ 3 per day $=9 ; 4-10$ per day $=10 ;>10$ per day $=11$ ); and, seizure frequency after AED introduction (seizure-free $=1$, plus same classification as above).

The protocol and the informed consent were approved by the ethical committee of our university hospital.

All 28 patients had CT scan, which were revised by a neuroradiologist with expertise in NC. Most CT scans were done soon after patient was admitted to our hospital. The disease activity was classified as active (appears on CT as hypodense cyst without enhancement), transitional (there is a ring or nodular contrast enhancement), and inactive

Departamentos de Neurologia e *Radiologia, Universidade Estadual de Campinas (UNICAMP), Campinas SP, Brasil: ${ }^{1}$ Pós-graduanda; ${ }^{2}$ Professor Doutor.

Received 22 March 2002, received in final form 1 July 2002. Accepted 15 July 2002.

Dra. Marilisa M. Guerreiro - Departamento de Neurologia - FCM/UNICAMP - Caixa Postal 6111 - 13083-970 Campinas SP - Brasil.

E-mail: mmg@fcm.unicamp.br 
(calcified lesions on CT) based on the viability of the parasite as proposed by Carpio et al. ${ }^{6}$. When lesions in different stages were found in the same patient, they were classified according to the most active lesion detected (active > transitional $>$ inactive). Lesions on CT were grouped in different categories: number $(=5,>5)$ and stage (active plus transitional and inactive).

We used multivariate analysis of variance (MANOVA) and simple main effect and post-hoc pairwise comparison in order to assess whether the stage of parasitic infection (active-transitional and inactive) and the number of lesions ( $=5$ and $>5$ lesions) might have main or interactive effect on seizure frequency before and short-term after AED treatment.

\section{RESULTS}

Concerning the evolutionary stage of the parasite, 17 patients were at inactive phase, six at transitional phase and five children had active lesions on CT. Regarding number of lesions, 18 patients had five or less, and 10 patients had more than five lesions.

Seizure frequency before and short-term after AED is shown in Table 1.

Multivariate ANOVA showed that the seizure frequency before AED treatment was not significantly affected by the stage of parasitic infection $[F(1,27)=0.23, p=0.638]$ or the number of lesions $[F(1,27)=1.82, p=0.19]$, or interaction between stage of parasitic infection and number of lesions $[F(1,27)=1.89, p=0.182]$.

Multivariate ANOVA showed that short-term seizure frequency after the AED treatment was not significantly affected by the stage of parasitic infection $[F(1,27)=1.59, p=0.219]$ or the number of lesions $[F(1,27)=2.04, p=0.166]$. However, there was a signi- ficant interaction between stage of parasitic infection and number of lesions $[F(1,27)=7.35, p=0.012]$. Simple main effect analysis, seeking the significant factor in the interaction between the stage of parasitic infection and number of lesions on seizure frequency, showed that the stage of parasitic infection had no significant effect on patients with $=5$ lesions $[F(1,24)=1.05, p=0.315]$, but had a significant effect on patients with $>5$ lesions $[F(1,24)=7.89, p=0.009]$. Simple main effect analysis of number of lesions with respect to the seizure frequency from different stage of the parasitic infection showed that number of lesions had significant effect on patients with activetransitional stage of parasitic infection $[F(1,24)=8.56$, $p=0.007]$, but not on patients with inactive stage of parasitic infection $[F(1,24)=0.82, p=0.373]$.

\section{DISCUSSION}

Neuroimaging has attained enormous progress during last decade. Despite that, $\mathrm{CT}$ is very helpful in NC because it is a safe, precise and noninvasive method with more than $95 \%$ accuracy to define number, localization and evolutionary stage of the parasite, especially in the parenchymal form of the disease ${ }^{7}$. In developing countries where MRI machines are not always available, and considering the fact that calcifications are the main radiological finding in NC, $\mathrm{CT}$ is still the most performed and useful examination.

Epilepsy is the most common clinical manifestation in NC. It occurs in $70-90 \%$ of all children with the parenchymal form of the disease, usually being its primary presentation ${ }^{7,8}$. The pathophysiology of the seizures due to $\mathrm{NC}$ is not completely understood

Table 1. Seizure frequency before and short-term after AED.

\begin{tabular}{llcccc}
\hline & & Before & Before & After & After \\
& & AED & AED & AED & AED \\
\hline \multirow{2}{*}{ Stage } & & 5 lesions & $>5$ lesions & 5 lesions & $>5$ lesions \\
Active and transitional & mean & 5.83 & 7.6 & 3.67 & 7 \\
& SD & 1.6 & 0.89 & 1.03 & 1.41 \\
& minimum & 5 & 7 & 3 & 5 \\
Inactive & maximum & 9 & 9 & 5 & 9 \\
& sample & 6 & 5 & 6 & 5 \\
& mean & 6.42 & 6.4 & 4.83 & 3.8 \\
& SD & 1.68 & 1.95 & 2.21 & 2.68 \\
& minimum & 5 & 5 & 2 & 2 \\
& maximum & 9 & 9 & 9 & 8 \\
& sample & 12 & 5 & 12 & 5 \\
\hline
\end{tabular}

*MANOVA showed significant interaction between stage of parasitic infection and number of lesions $[F(1,27)=7.35$, $p=0.012]$. See text for detailed statistical description. 
yet. In active and transitional forms, seizures may be the consequence of compression or inflammatory reaction. In inactive form, perilesional gliosis is probably the cause of the seizures. Chronic inflammatory reaction sometimes takes several years to disappear and it may have an important role in the pathophysiology of focal epilepsy in $\mathrm{NC}^{9,10}$.

Parenchymal NC is the most frequent form in children $^{7}$. In this age group, the disease behaves differently from adults in several aspects, which have important implications in diagnosis, management and prognosis. Massive infestation and multiple lesions, for example, occur more frequently in children than adults².

In this study, we demonstrated that there was an interaction between number and viability of the parasites on seizure frequency after AED introduction. Our data showed that patients with $>5$ lesions in active and transitional stages had higher seizure frequency after AED introduction, predicting a worse prognosis. The explanation may be that NC in children has a peculiar behavior. A massive infestation by the eggs of Taenia solium, which develop to viable cysts representing the larval form of the disease, may occur in this age group. For a variable period of time these cysts remain unchanged and exhibit a state of immune tolerance with the host. Patients may have seizures, or may remain asymptomatic. Eventually the cysts die and dying cysts have a much greater likelihood of being symptomatic ${ }^{9}$. Dying cysts at different times may cause successive periods of exacerbation of symptoms. This is a dynamic process that may take years to resolve completely and may lead to recurrence of seizures.

\section{REFERENCES}

1. WhiteAC Jr. Neurocysticercosis: a major cause of neurological disease worldwide. Clin Infect Dis 1997;101-115.

2. Sotelo J, Del Bruto OH. Brain cysticercosis: review article. Arch Med Res 2000;31:3-14.

3. Monteiro L, N unes B, Mendonça D. Spectrum of epilepsy in neurocysticercosis: a long term follow up of 143 patients. Acta N eurol Scand 1995;92:33-40.

4. Del Bruto OH, Wadia NH, Dumas M, Tsang VCW, Schantz P.M. Proposal of diagnostic criteria for human cysticercosis and neurocysticercosis. J Neurol Sci 1996;142:1-6.

5. Engel J Jr, Van Ness PC, Rasmussen TB, Ojemann LM. Outcome with respect to epileptic seizures In: Engel J Jr, (Ed.) Surgical treatment of the Epilepsies, 2.Ed. New York: Raven Press, 1993:609-621.

6. Carpio A, Marcelo P, Santillan F, Alfonso E. A proposal for classification of neurocysticercosis. Canj Neurol Sci 1994;21:43-47.

7. Garcia MR, Astiazarán AG, Franco FR. Neurocysticercosis in children clinical experience in 122 patients. Child's Nerv Syst 1997;13:608-612.

8. Bittencourt PRM, Adamolekum B, Bharucha N, et al. Epilepsy in tropics: II. Clinical presentations, pathophisiology, immunologic diagnosis, economics, and therapy. Epilepsia 1996;37:1128-1137.

9. Carpio A, Escobar A, Hauser WA. Cysticercosis and epilepsy: a critical review. Epilepsia 1998;39:1025-1040.

10. Del Bruto OH, Santibãnes R, N oboa CA, Aguirre R, Diaz R, Alarcón TA. Epilepsy due to neurocysticercosis: analysis of 203 patients. Neurology 1992;42:389-392. 